\title{
Motor skills in autism: A missed opportunity
}

\author{
BY ASHLEY DE MARCHENA, CASEY ZAMPELLA
}

4 JANUARY 2022

\section{Listen to this story:}

https://www.spectrumnews.org/wp-

content/uploads/2022/01/audio-2495f9af-2b0f-4c34-a19e-08b3ffccecf7-encodings.mp3

Eating a delicious meal, ranting to a friend, playing an instrument, tackling that boulder scramble in the middle of your favorite hike - some of life's greatest pleasures depend on a diverse and complex set of motor skills. Despite the centrality of motor function to so many aspects of everyday living, its connection to many neurodevelopmental and mental health conditions is poorly understood. And overlooking its link to autism has led to missed opportunities for both research and clinical care.

Clinicians and scientists have described motor skill differences since the earliest conceptualizations of autism, yet these differences are widely viewed as peripheral to the condition's core traits. In the Diagnostic and Statistical Manual of Mental Disorders, 5th Edition (DSM-5), for instance, atypical gait and clumsiness are included only as 'associated features,' independent of the primary phenotype.

Accordingly, autism theories, assessments and interventions have tended to gloss over the extent to which motor skill differences affect autistic people. A burgeoning literature, however, suggests that broad motor differences are more relevant than has historically been appreciated for understanding, assessing and supporting people on the spectrum.

We recently reviewed the most current research on motor skills in autism, with a focus on the evidence most relevant for clinical care. We concluded that motor skill differences are a meaningful and highly underutilized clinical target for autistic people of all ages. They should also be included in the DSM as a clinical specifier for autism. Such representation would signal the need to give targeted attention to motor functioning, and it would provide a clear framework for how motor 


\section{Spectrum | Autism Research News}

https://www.spectrumnews.org

differences fit into the broader diagnostic picture.

Autistic and non-autistic people show large group-level differences across various motor skill areas, according to numerous recent reviews and meta-analyses. Three large-scale prevalence studies have also confirmed that general motor differences are pervasive, clinically significant and underrecognized in autism. Up to 87 percent of autistic children exhibit motor challenges, yet only a small number receive a motor-specific diagnosis (15 percent) or treatment (32 percent), revealing a considerable clinical gap. Based on these findings, motor challenges are at least as prevalent in people with autism as either cognitive or language impairment, which are both DSM specifiers and widely thought to shape individual presentations, treatment recommendations and outcomes.

Given the pervasiveness of motor differences in autism, it is possible that they are not simply associated with the diagnosis but inherently related to core autism traits. Developmentally, motor skills play a key role in shaping children's interactions with other people and their environments from infancy, and are thus intrinsically linked to social, communication, adaptive and cognitive skill development. The first communication skills to emerge — facial expressions, joint attention and protoimperative pointing - are all motor behaviors. Early differences in motor behavior could therefore have cascading developmental effects across domains.

Indeed, early fine and gross motor skills and both concurrent and future communication skills in young children who are ultimately diagnosed with autism are broadly associated, according to a meta-analysis. Similarly, among older children, greater risk for motor impairment is tied to socialcommunication skills and restricted and repetitive behaviors, two recent studies of the nationally representative SPARK dataset suggest.

Social-communication cues such as eye contact, facial expressions, social orienting and gestures all fundamentally rely on movement, and even subtle atypicalities in these nonverbal cues can alter their effectiveness. The global ways in which people move their bodies (including gait, posture and coordination) are also highly salient to others in everyday contexts. As a result, challenges with motor planning and execution could directly influence social interactions and social perceptions of autistic people.

Researchers have begun to theorize that basic differences in how autistic and non-autistic people move may make it more difficult for them to connect, reflective of the "double-empathy" problem. Our own recent work suggests that autistic children and adolescents are less likely than their neurotypical peers to synchronize body movements and facial expressions with those of neurotypical conversation partners, and that this reduced social coordination is associated with more pronounced autism traits and less typical social and communication skills.

Of course, the impact of motor skill differences extends far beyond social communication. Difficulty or discomfort with movement-based activities may prevent autistic children and adults from 


\section{Spectrum | Autism Research News}

https://www.spectrumnews.org

engaging in healthy, pleasurable activities such as exercise, artistic hobbies or even meals. One of us (de Marchena) worked on a treatment study for selective eating in autistic children and distinctly remembers a 10-year-old who would eat only finger foods. Although it is often assumed that selective eating is due to cognitive inflexibility or sensory aversions, this boy's father was convinced that his son's was driven in large part by his discomfort and frustration with using utensils.

Treating fundamental motor difficulties might help with problems misinterpreted as oppositional behavior. For example, a child with autism may actively resist getting ready for school because delayed fine motor skills make daily tasks such as tying shoes or buttoning a coat particularly challenging. Directly supporting motor skill differences may provide a novel path for improving functional outcomes, and reducing frustration, across a variety of daily-living domains.

Also, autistic adults who are not interested in interventions focused on changing their core autism traits might welcome motor-focused ones that could improve their functional living skills or quality of life. Motor interventions in autism are receiving increased empirical attention, with promising improvements seen in motor skills themselves and in downstream social skills for some children, though much of the research to date consists of small pilot or non-controlled studies.

More explicit recognition of the scope and relevance of motor differences in autism, such as in the DSM, would prompt increased interest and funding for comprehensive intervention studies in this area. It might also have significant implications for early detection and assessment. Motor skills may be the domain in which developmental divergence appears first. Infants later diagnosed with autism show fine and gross motor differences by just 6 months of age, and large group-level differences are observed by 13 months of age. Recognizing that motor differences may precede the onset of core autism behaviors could also shed light on the condition's developmental pathways.

An increased awareness of just how often autism and motor conditions, such as developmental coordination disorder, co-occur could also prompt clinicians to incorporate simple motor screening and evaluation into routine practice. Brief, freely available parent-report screeners, for instance, can identify motor impairment and co-occurring motor conditions in autistic children, providing an important opportunity to connect people with relevant services.

Many theoretical questions remain about the specificity of motor disruption to autism, and these should be the focus of further cross-diagnostic and multidimensional research. For example, which motor differences in infancy are specific markers of autism, and which reflect more general developmental delays? Our research team is particularly interested in whether there are distinct motor profiles unique to autism that can be parsed using computational approaches.

We also support continued, larger-scale research on motor skill interventions that may have a meaningful influence on functioning. Although we speculate that this could be a beneficial form of 


\section{Spectrum | Autism Research News}

https://www.spectrumnews.org

intervention for some autistic people, we are not yet aware of any research specifically asking autistic people about how motor skill differences affect their lives and what, if any, supports would appeal to them - work that needs to be done.

We strongly advocate looking beyond interventions that place the burden of change on autistic people, and broader modifications that make existing resources more accessible to people with motor differences. Physical activity, in particular, benefits both physical and mental health, and reducing barriers for all people is essential to optimal life outcomes. The "Hiking is for everyone" guidelines, for example, coach caregivers on how to support hikers with disabilities and illustrate how an increased awareness of different motor needs can go a long way toward increasing inclusivity.

Ashley de Marchena is assistant professor of psychology and neuroscience at the University of the Sciences in Philadelphia, Pennsylvania. Casey Zampella is a scientist at the Center for Autism Research at Children's Hospital of Philadelphia.

Cite this article: https://doi.org/10.53053/VRWB1457 